\title{
A Monte-Carlo Study on the Coupling of Magnetism and Ferroelectricity in the Hexagonal Multiferroic $\mathrm{RMnO}_{3}$
}

\author{
Chunruo Duan, Gia-Wei Chern and Despina Louca * \\ Physics Department, University of Virginia, Charlottesville, VA 22904, USA; cd4fj@virginia.edu (C.D.); \\ gc6u@virginia.edu (G.-W.C.) \\ * Correspondence: louca@virginia.edu; Tel.: +1-434-924-6802
}

Received: 15 August 2018; Accepted: 18 September 2018; Published: 20 September 2018

\begin{abstract}
The ferroelectric phase transition in $\mathrm{RMnO}_{3}$ breaks both $\mathrm{Z}_{3}$ and $\mathrm{Z}_{2}$ symmetries, giving rise to 6 structural domains. Topological protected vortices are formed at the junctions of all 6 domains, and the ferroelectric phase transition is closely related to these $Z_{6}$ vortices. In this work, Monte-Carlo studies on both the ferroelectric and magnetic transition have been performed on $\mathrm{RMnO}_{3}$ system. The magnetic simulation results on lattices with different structural domain distributions induced by external electric field and simulated quenching show different magnetic transition temperature $T_{S}$, indicating that the coupling of magnetism and ferroelectricity is through the $\mathrm{Z}_{6}$ structural domain. At extreme case, lattice quenched from above the ferroelectric transition results in high vortex density, which can drive the system into spin glass.
\end{abstract}

Keywords: multiferroics; Monte-Carlo simulation; $Z_{6}$ symmetry breaking

Multiferroics are materials that host magnetism and ferroelectricity in a single phase [1]. The potential of having magnetism and ferroelectricity coupling makes these materials of great importance both technologically and scientifically. Judging by the source of magnetism and ferroelectricity, multiferroics can be categorized into type-I and type-II: in type-I multiferroics the magnetism and ferroelectricity have different origins and are often well separated in transition temperatures, whereas in type-II multiferroics the magnetism is the cause of ferroelectricity [2-4]. As a typical type-I multiferroic, the hexagonal $\mathrm{RMnO}_{3}(\mathrm{R}=\mathrm{Y}, \mathrm{Lu}, \mathrm{Sc}, \cdots)$ becomes ferroelectric below $T_{C} \sim 570-990 \mathrm{~K}$ when the crystal breaks the $Z_{3}$ symmetry of the high temperature $P 6_{3} m m c$ phase through a structural instability of the $\mathrm{Mn}$ and $\mathrm{O}$ atoms due to trimerization [5-7]. The trimerization then leads to the rare earth atom splitting into two atomic sites which breaks the $Z_{2}$ symmetry and gives rise to polarization, as illustrated in Figure $1 \mathrm{~b}$. The space group of the ferroelectric phase is $\mathrm{P}_{3} \mathrm{~cm}$ [8]. Since the $\mathrm{Mn}$ atoms form triangular lattice in the xy-plane, the system becomes geometrically frustrated [9]. Below $\mathrm{T}_{N} \sim 90 \mathrm{~K}$, the magnetic moment of Mn orders in a $120^{\circ}$ arrangement $[8,10]$. The weak interaction between magnetism and ferroelectricity in $\mathrm{RMnO}_{3}$ was observed through in-plane dielectric anomaly [11].

The $Z_{3} \times Z_{2}$ symmetry breaking in the ferroelectric transition of $\mathrm{RMnO}_{3}$ creates 6 structural domains. As shown in Figure $1 \mathrm{a}-\mathrm{c}, \mathrm{RMnO}_{3}$ consists of corner sharing $\mathrm{MnO}_{5}$ bipyramid layers separated by the rare earth atom layers. From the top view, each $\mathrm{MnO}_{5}$ bipyramid is surrounded by 3 rare earth atoms $(\alpha, \beta, \gamma)$. The trimerization involves tilting of the bipyramids towards one of the 6 symmetry equivalent directions: $\alpha^{+}, \beta^{+}, \gamma^{+}, \alpha^{-}, \beta^{-}, \gamma^{-}$, each of which corresponds to a structural domain [6]. This has been confirmed by observations using optics second harmonic generation, SEM, STM and AFM [6,12-16]. Both the magnetic and ferroelectric order parameters are confined in the xy-plane due to the crystal geometry [17]. Based on this feature, a 2D 6-fold clock model without 
microscopical details can be built for Monte-Carlo study of $\mathrm{RMnO}_{3}$ system [18]. Without external electric field, the Hamiltonian of the model has the following form:
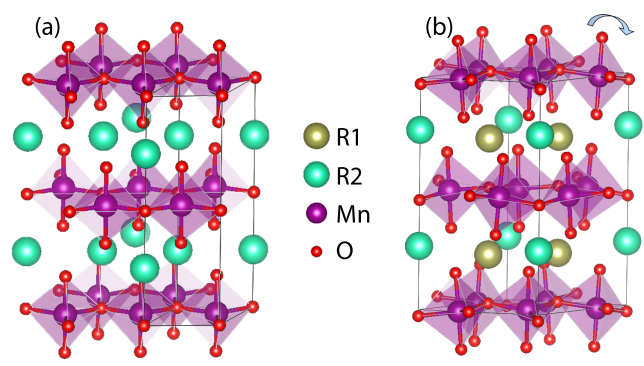

(c)

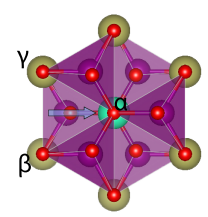

(d)

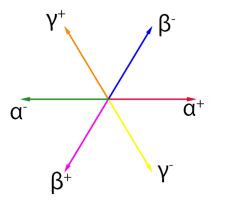

(e)

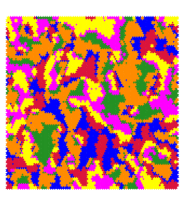

Figure 1. (Color online.) Shown in $(\mathbf{a}, \mathbf{b})$ are the crystal structures of $\mathrm{RMnO}_{3}$ above and below the ferroelectric transition temperature $T_{C}$, respectively. The arrow in $(\mathbf{b})$ indicates the direction of the $\mathrm{MnO}_{5}$ bipyramid tilting (a.k.a. trimerization). In (c) shows the 6-symmetry equivalent trimerization directions, which correspond to the 6 structural domains as labeled in (d). Shown in (e) is a typical simulated lattice below $\mathrm{T}_{C}$.

$$
H_{E}=-\sum_{i} A_{E} \cos \left(6 \varphi_{i}\right)-\sum_{\langle i, j\rangle} J_{E} \cos \left(\varphi_{i}-\varphi_{j}\right)
$$

Here $\varphi_{i}$ represents direction of the bipyramid tilting on the $i$ th unit cell, $A_{E}$ is the structural anisotropy coefficient, and $J_{E}$ is the coupling constant between the nearest neighbor $\langle i, j\rangle$. To simulate the hexagonal crystal structure of $\mathrm{RMnO}_{3}$, the Hamiltonian is defined on a triangular lattice. The first term determines 6 minima of the simulated variable: $\varphi_{n}=\frac{n \pi}{3}$ with $n=0,1, \cdots, 5$, each corresponding to one of the 6 structural domains, as illustrated in Figure 1d. The second term introduces ferroelectric coupling between nearest neighbors with positive value of $J_{E}$. The Metropolis Monte-Carlo algorithm is used to simulate the system. In each iteration, a unit cell $i$ is chosen randomly. A random change $d \varphi$ is proposed to be added on $\varphi_{i}$, and will be accepted with probability $\operatorname{MIN}(1, \exp (-\beta \Delta E))$, with $\Delta E$ being the corresponding change of the total energy and $\beta=\frac{1}{k_{B} T}$. A typical simulated lattice in the ferroelectric phase is shown in Figure 1e, where different domains are painted in different colors. The domain walls always cross at the junctions of all 6 domains, forming $Z_{6}$ vortices, which are direct results of the $Z_{3} \times Z_{2}$ symmetry breaking. In the simulation the vortices always appear in two low-energy cyclic sequences: $\alpha^{+}, \beta^{-}, \gamma^{+}, \alpha^{-}, \beta^{+}, \gamma^{-}$and $\alpha^{+}, \gamma^{-}, \beta^{+}, \alpha^{-}, \gamma^{+}, \beta^{-}$, in agreement with the experimental observations $[13,15,16]$. In $3 \mathrm{D}$ the vortices in each $x y$-plane are connected, forming vortex loops [17,18].

The order parameter of this $Z_{6}$ symmetry breaking transition is defined as:

$$
m_{Z_{6}}=\frac{1}{N}\left\{\left[\sum_{i} \cos \left(\varphi_{i}\right)\right]^{2}+\left[\sum_{i} \sin \left(\varphi_{i}\right)\right]^{2}\right\}^{1 / 2}
$$

If the variable $\varphi$ goes through $2 \pi$ or $-2 \pi$ across the 6 nearest neighbors of a site $i$, this site is classified as a vortex with positive or negative cyclic sequence. With this definition, the high temperature phase consists of high density of vortices. The polarization per site of the simulated lattice is calculated by:

$$
P=p_{0} \sum_{i} \cos \left(3 \varphi_{i}\right) / N
$$

Here $p_{0}$ is the unit dipole moment of a unit cell. With that the Hamiltonian with external electric field can be defined as: 


$$
\begin{aligned}
H_{E}= & -\sum_{i} A_{E} \cos \left(6 \varphi_{i}\right)-\sum_{\langle i, j\rangle} J_{E} \cos \left(\varphi_{i}-\varphi_{j}\right) \\
& -E \cdot p_{0} \sum_{i} \cos \left(3 \varphi_{i}\right) / N
\end{aligned}
$$
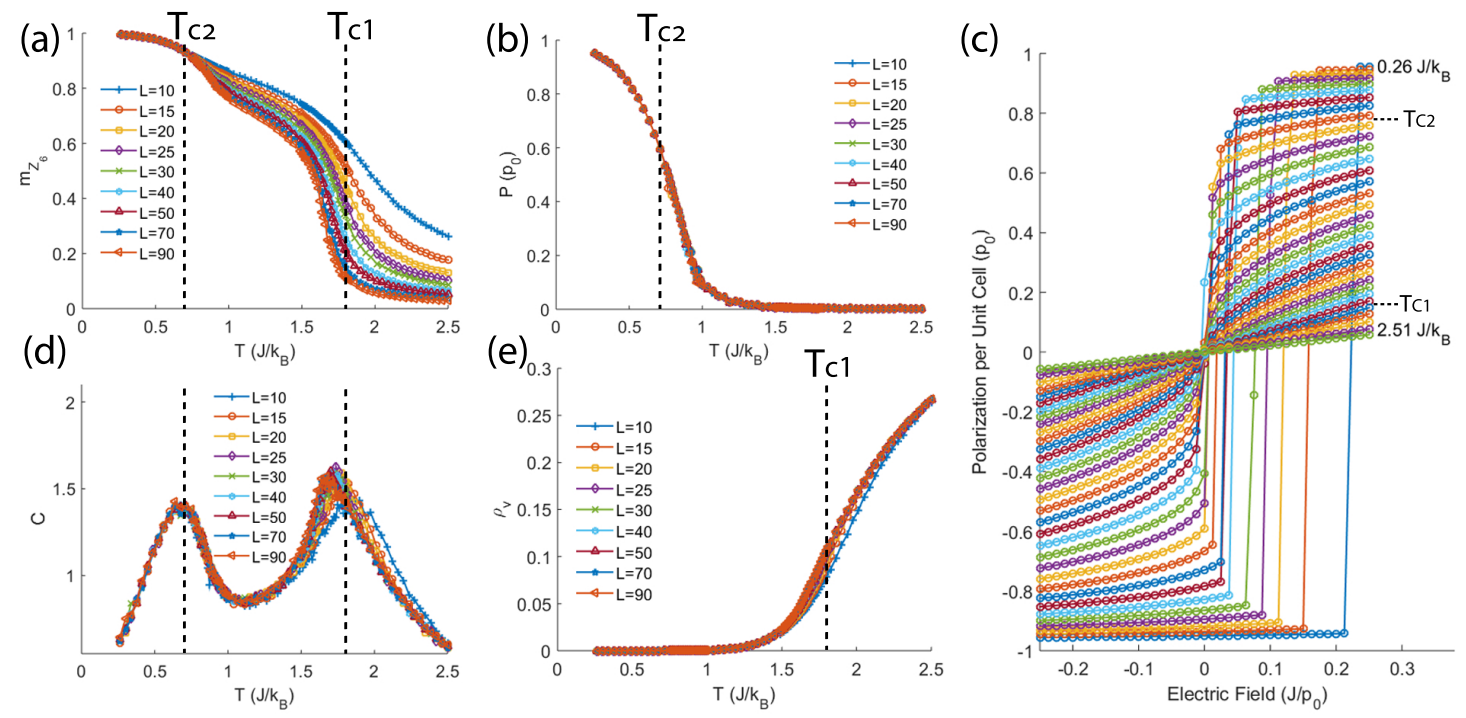

Figure 2. (Color online.) Above shows the simulation results on the ferroelectric part of the model. First, the ferroelectric transition with different lattice sizes is simulated under zero electric field. The $\mathrm{Z}_{6}$ order parameter $m_{Z_{6}}$, the polarization $P$, the heat capacity $C$ and the density of $Z_{6}$ vortices $\rho_{v}$ are plotted as functions of simulated temperature in $(\mathbf{a}, \mathbf{b}, \mathbf{d}, \mathbf{e})$, respectively. Second, the responds of an $L=40$ lattice to the external electric field in temperature range of $0.26 \sim 2.51 \mathrm{~J} / k_{B}$ are demonstrated in (c).

The ferroelectric transition with different lattice sizes is simulated under zero electric field with $J_{E}=J, A_{E}=\frac{5}{8} J$. The $Z_{6}$ order parameter $m_{Z_{6}}$, the polarization $P$, the heat capacity $C$ and the density of $Z_{6}$ vortices $\rho_{v}$ are plotted as functions of simulated temperature in Figure $2 \mathrm{a}, \mathrm{b}, \mathrm{d}, \mathrm{e}$, respectively. The simulation clearly showed two transitions: $\mathrm{T}_{C 1} \sim 1.8 \mathrm{~J} / k_{B}$ marks the temperature where the vortex density drops, and $\mathrm{T}_{\mathrm{C} 2} \sim 0.7 \mathrm{~J} / k_{\mathrm{B}}$ is associated with the onset of polarization. It is known that a $2 \mathrm{D}$ xy model does not yield any 1st or 2nd order phase transition, but can give rise to the Kosterliz-Thouless (KT) transition [19]. Previous theoretical works have shown that the 2D 6-state clock model can go from disorder to order through either an Ising transition plus a 3-state Potts transition or two KT transitions [20]. Numerical study on systems with similar $Z_{6}$ symmetry breaking shows evidence of $2 \mathrm{KT}$ transitions with a critical phase in between [21,22]. As shown in Figure $2 \mathrm{a}, \mathrm{b}, \mathrm{d}, \mathrm{e}$, the two transitions we observed exhibit little lattice-size dependence. Finite size scaling analysis on these two transitions supports neither Ising transition nor Potts transition. Therefore the two ferroelectric transitions in our model are most likely KT transitions. Shown in Figure 2c are the simulation results of polarization as a function of the electric field $\mathrm{E}$ in a temperature range of $0.26 \sim 2.51 \mathrm{~J} / k_{B}$ on a $L=40$ lattice. Initially the lattice was fully polarized by negative field. The external electric field was then gradually increased. The simulated lattice reached equilibrium at each field point. Below $T_{C 2}$ the system shows a clear ferroelectric hysteresis. Between $T_{C 2}$ and $T_{C 1}$ the system shows paraelectric behavior with a non-linear P-E curve, similar to the superparaelectric P-E curve observed in relaxor ferroelectrics [23,24]. Above $T_{C 1}$ the system becomes a normal paraelectric with linear P-E curve. The superparaelectric behavior between $T_{C 2}$ and $T_{C 1}$ suggest that the system is in a critical phase with infinite correlation length, which further supports that the two observed transition is KT type. The results of the simulation on the ferroelectric variable with zero electric field 
demonstrate that the $Z_{6}$ symmetry breaking in our model is achieved through two KT transitions. First the system breaks $Z_{3}$ symmetry, indicated by the decrease of vortex density, then the system breaks $\mathrm{Z}_{2}$ symmetry, leading to non-zero polarization. This two-step nature of the ferroelectric transition in $\mathrm{RMnO}_{3}$ has been observed experimentally [25]. The lattice responds to external electric field is in agreement with experimental results $[15,16,26]$.

On top of the 6-fold clock model for ferroelectricity of the system, a 2D xy model for spin order can be added by introducing the following Hamiltonian [27]:

$$
H_{S}=-\sum_{i} A_{S} \cos \left(2 \phi_{i}\right)-\sum_{\langle i, j\rangle} J_{S} \cos \left(\psi_{i}-\psi_{j}\right)
$$
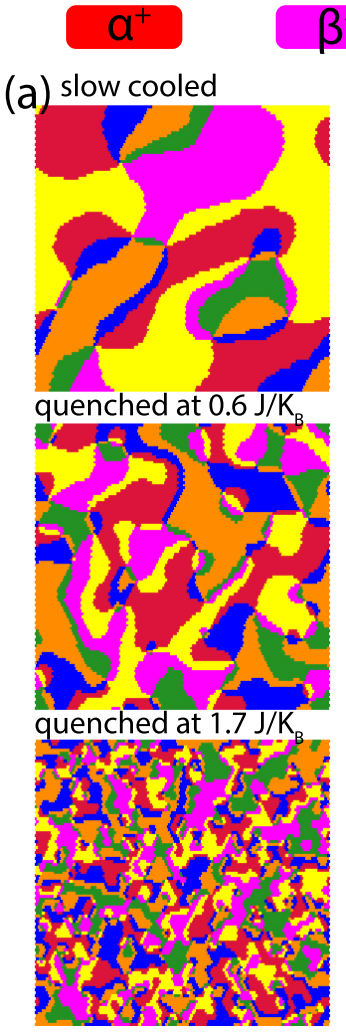

\section{$\beta^{+}$} polarized

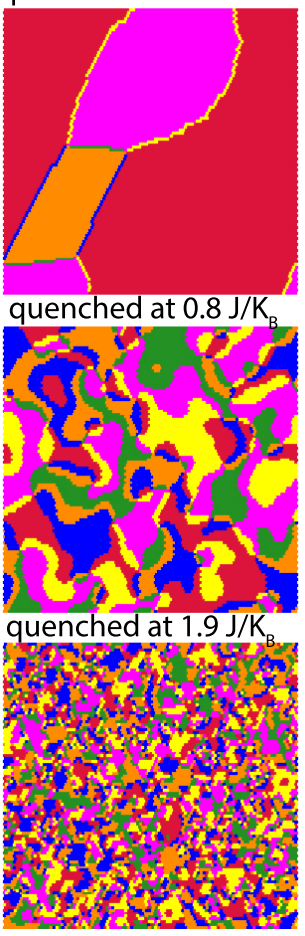

\section{$a^{-}$}

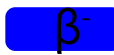

Y

(b)
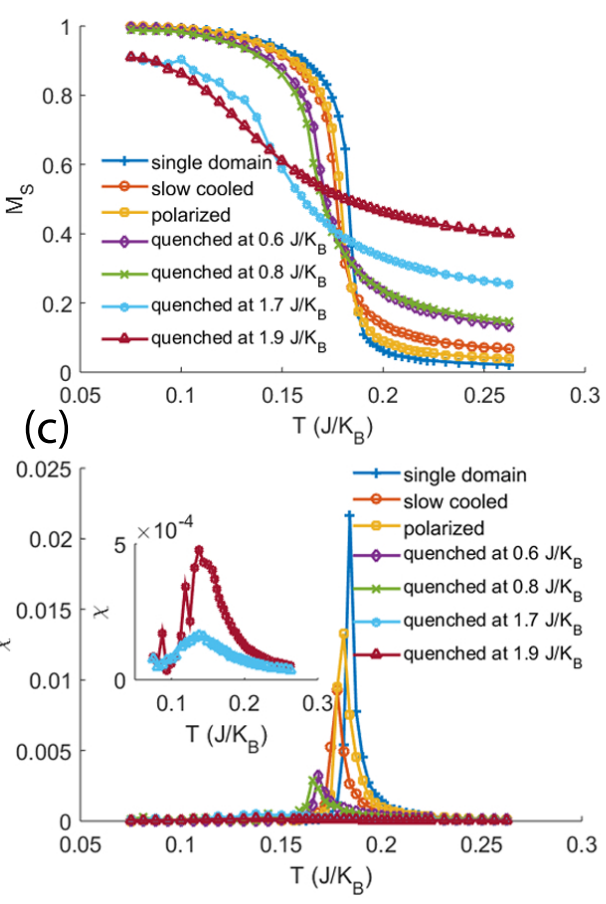

Figure 3. (Color online.) Shown in (a) are six $100 \times 100$ simulated lattices with different structural domain distributions. The magnetic order parameters and susceptibilities of the six lattices plus a single domain case are plotted in $(\mathbf{b}, \mathbf{c})$ as functions of temperature. The inset in (c) are zoomed-in plot of the susceptibility curves of the last two quenched lattices.

Here the variable $\phi_{i}$ represents the magnetic ordering within the $i$ th unit cell, and the variable $\psi_{i} \equiv \varphi_{i}+\phi_{i}$ takes the ferroelectric domain of that unit cell into account. $A_{S}$ represents the single-ion anisotropy for spin, and $J_{S}$ is the coupling constant between the nearest neighbor $\langle i, j\rangle$. The magnetization per site in the $j$ th structural domain is calculated by:

$$
M_{j}=\frac{\mu_{0}}{N_{j}}\left\{\left[\sum_{i} \cos \left(\phi_{i}\right)\right]^{2}+\left[\sum_{i} \sin \left(\phi_{i}\right)\right]^{2}\right\}^{1 / 2}
$$

with $\mu_{0}$ representing the magnetic moment within a unit cell, and $N_{j}$ being the size of the $j$ th domain. If fixing the ferroelectric variable $\varphi_{i}=0$ across the whole lattice, the spin part of the model is equivalent 
to a 2D Ising model under strong anisotropy term $A_{S}$. By averaging among different structural domains the magnetic order parameter of the whole lattice is defined as:

$$
M_{S}=\frac{1}{N} \sum_{j} N_{j} M_{j}
$$

With only one structural domain, the magnetic order parameter is Ising like with reasonably large anisotropy $A_{S}$. It is then interesting to study how the magnetic transition behaves when the lattice exhibits different level of complexity in structural domain distribution. Since the ferroelectric transition temperature of $\mathrm{RMnO}_{3}$ is an order of magnitude larger than its magnetic transition temperature, the value of the parameters in $H_{S}$ were chosen to be $A_{S}=J_{S}=0.05 \mathrm{~J}$. Shown in Figure 3a are six $100 \times 100$ simulated lattices with different structural domain distributions. The 'slow cooled' lattice was simulated by gradually decreasing the temperature from above $T_{C 1}$ to $0.1 \mathrm{~J} / k_{B}$. The 'quenched' lattices were simulated by abruptly changing the temperature from the labeled values to $0.1 \mathrm{~J} / \mathrm{k}_{B}$. The 'polarized' lattice was created by applying positive electric field on the 'slow cooled' lattice. The simulation results of the magnetic order on these lattices plus a trivial single domain case are shown in Figure $3 b, c$ in the form of magnetization and magnetic susceptibility. With increased structural domain complexity and decreased average domain size, the magnetic moments order with a lower transition temperature and a lower saturated magnetization at base temperature. Previous works have shown coupling between magnetoelastic coupling in this system using inelastic neutron scattering and ultrasonic measurements $[28,29]$. In our model, the only thing that couples the magnetism and ferroelectricity is the $\sum_{\langle i, j\rangle} J_{S} \cos \left(\psi_{i}-\psi_{j}\right)$ term in Equation (3), where the first nearest neighbor coupling of the magnetic moment is weakened if the two neighbors belong to different ferroelectric domains. When the structural domain becomes more complex, the frequently changing $\varphi_{i}$ induces disorder in the coupling constant $J_{S}$, which drives the system towards the Edwards-Anderson model for spin glass [30]. In fact, the magnetic susceptibility curves of the 'quenched at $1.7 \mathrm{~J} / k_{B}$ ' and 'quenched at $1.9 \mathrm{~J} / \mathrm{k}_{B}{ }^{\prime}$ lattices shown in the inset of Figure $3 \mathrm{c}$ is very similar to the no-field-cooling curve of a spin glass. In conclusion, our Monte-Carlo simulation work demonstrates that the magnetism and the ferroelectricity in the type-I multiferroic material $\mathrm{RMnO}_{3}$ can still be coupled through the $\mathrm{Z}_{6}$ structural domains, and that in extreme case the high vortex density can drive the system into a spin glass state.

Author Contributions: Conceptualization, D.L., G.-W.C. and C.D.; methodology, G.-W.C.; data curation, C.D.; formal analysis, C.D.; writing—original draft, C.D.; writing—review \& editing, G.-W.C., D.L.; supervision, D.L.

Funding: This research was funded by the US Department of Energy, Office of Basic Energy Science, DE-FG02-01ER45927.

Conflicts of Interest: The authors declare no conflict of interest.

\section{References}

1. Spaldin, N.A. MATERIALS SCIENCE: The Renaissance of Magnetoelectric Multiferroics. Science 2005, 309, 191-192. [CrossRef] [PubMed]

2. Kimura, T.; Goto, T.; Shintani, H.; Ishizaka, K.; Arima, T.; Tokura, Y. Magnetic Control of Ferroelectric Polarization. Nature 2003, 426, 55-58. [CrossRef] [PubMed]

3. Hur, N.; Park, S.; Sharma, P.A.; Ahn, J.S.; Guha, S.; Cheong, S.W. Electric Polarization Reversal and Memory in a Multiferroic Material Induced by Magnetic Fields. Nature 2004, 429, 392-395. [CrossRef] [PubMed]

4. Khomskii, D. Classifying Multiferroics: Mechanisms and Effects. Physics 2009, 2, 20. [CrossRef]

5. Yakel, H.L., Jr.; Koehler, W.C.; Bertaut, E.F.; Forrat, E.F. On the Crystal Structure of the Manganese(III) Trioxides of the Heavy Lanthanides and Yttrium. Acta Crystallograph. 1963, 16, 957-962. [CrossRef]

6. Choi, T.; Horibe, Y.; Yi, H.T.; Choi, Y.J.; Wu, W.; Cheong, S.W. Insulating Interlocked Ferroelectric and Structural Antiphase Domain Walls in Multiferroic $\mathrm{YMnO}_{3}$. Nat. Mater. 2010, 9, 253-258. [CrossRef] [PubMed] 
7. Aikawa, Y.; Katsufuji, T.; Arima, T.; Kato, K. Effect of Mn Trimerization on the Magnetic and Dielectric Properties of Hexagonal $\mathrm{YMnO}_{3}$. Phys. Rev. B 2005, 71, 184418. [CrossRef]

8. Tomuta, D.G.; Ramakrishnan, S.; Nieuwenhuys, G.J.; Mydosh, J.A. The Magnetic Susceptibility, Specific Heat and Dielectric Constant of Hexagonal $\mathrm{YMnO}_{3}, \mathrm{LuMnO}_{3}$ and $\mathrm{ScMnO}_{3}$. J. Phys. Condens. Matter 2001, 13, 4543-4552. [CrossRef]

9. Katsufuji, T.; Mori, S.; Masaki, M.; Moritomo, Y.; Yamamoto, N.; Takagi, H. Dielectric and Magnetic Anomalies and Spin Frustration in Hexagonal $\mathrm{RMnO}_{3}(\mathrm{R}=\mathrm{Y}, \mathrm{Yb}$, and Lu). Phys. Rev. B 2001, 64, 104419. [CrossRef]

10. Muñoz, A.; Alonso, J.A.; Martínez-Lope, M.J.; Casáis, M.T.; Martínez, J.L.; Fernández-Díaz, M.T. Magnetic Structure of Hexagonal $\mathrm{RMnO}_{3}(\mathrm{R}=\mathrm{Y}$, Sc): Thermal Evolution From Neutron Powder Diffraction Data. Phys. Rev. B 2000, 62, 9498-9510. [CrossRef]

11. Tian, W.; Tan, G.; Liu, L.; Zhang, J.; Winn, B.; Hong, T.; Fernandez-Baca, J.A.; Zhang, C.; Dai, P. Influence of Doping on the Spin Dynamics and Magnetoelectric Effect in Hexagonal $\mathrm{Y}_{0.7} \mathrm{Lu}_{0.3} \mathrm{MnO}_{3}$. Phys. Rev. B 2014, 89, 144417. [CrossRef]

12. Fiebig, M.; Lottermoser, T.; Fröhlich, D.; Goltsev, A.V.; Pisarev, R.V. Observation of Coupled Magnetic and Electric Domains. Nature 2002, 419, 818-820. [CrossRef] [PubMed]

13. Li, J.; Yang, H.X.; Tian, H.F.; Ma, C.; Zhang, S.; Zhao, Y.G.; Li, J.Q. Scanning Secondary-Electron Microscopy on Ferroelectric Domains and Domain Walls in $\mathrm{YMnO}_{3}$. Appl. Phys. Lett. 2012, 100, 152903. [CrossRef]

14. Lottermoser, T.; Lonkai, T.; Amann, U.; Hohlwein, D.; Ihringer, J.; Fiebig, M. Magnetic Phase Control by an Electric Field. Nature 2004, 430, 541-544. [CrossRef] [PubMed]

15. Chae, S.C.; Lee, N.; Horibe, Y.; Tanimura, M.; Mori, S.; Gao, B.; Carr, S.; Cheong, S.W. Direct Observation of the Proliferation of Ferroelectric Loop Domains and Vortex-Antivortex Pairs. Phys. Rev. Lett. 2012, 108, 167603. [CrossRef] [PubMed]

16. Chae, S.C.; Horibe, Y.; Jeong, D.Y.; Lee, N.; Iida, K.; Tanimura, M.; Cheong, S.W. Evolution of the Domain Topology in a Ferroelectric. Phys. Rev. Lett. 2013, 110, 167601. [CrossRef] [PubMed]

17. Rowley, S.E.; Lonzarich, G.G. Ferroelectrics in a Twist. Nat. Phys. 2014, 10, 907-908. [CrossRef]

18. Lin, S.; Wang, X.; Kamiya, Y.; Chern, G.W.; Fan, F.; Fan, D.; Casas, B.; Liu, Y.; Kiryukhin, V.; Zurek, W.H.; et al. Topological Defects as Relics of Emergent Continuous Symmetry and Higgs Condensation of Disorder in Ferroelectrics. Nat. Phys. 2014, 10, 970-977 [CrossRef]

19. Kosterlitz, J.M.; Thouless, D.J. Ordering, Metastability and Phase Transitions in Two-dimensional Systems. J. Phys. C Solid State Phys. 1973, 6, 1181. [CrossRef]

20. Cardy, J.L. General Discrete Planar Models in Two Dimensions: Duality Properties and Phase Diagrams. J. Phys. A Math. Gener. 1980, 13, 1507-1515. [CrossRef]

21. Chern, G.W.; Tchernyshyov, O. Magnetic Charge and Ordering in Kagome Spin Ice. Philos. Trans. R. Soc. Lond. A Math. Phys. Eng. Sci. 2012, 370, 5718-5737. [CrossRef] [PubMed]

22. Challa, M.S.; Landau, D.P. Critical Behavior of the Six-state Clock Model in Two Dimensions. Phys. Rev. B 1986, 33, 437-443. [CrossRef]

23. Skulski, R. Superparaelectric Behaviours of Relaxor Ferroelectrics. Mater. Sci. Eng. B 1999, 64, $39-43$. [CrossRef]

24. Cross, L.E. Relaxor Ferroelectrics. Ferroelectrics 1987, 76, 241-267. [CrossRef]

25. Skjærvø, S.H.; Meier, Q.; Feygenson, M.; Spaldin, N.A.; Billinge, S.J.L.; Bozin, E.S.; Selbach, S.M. Unconventional Order-disorder Phase Transition in Improper Ferroelectric Hexagonal Manganites. arXiv 2017, arXiv:1707.09649.

26. Wang, X.; Mostovoy, M.; Han, M.G.; Horibe, Y.; Aoki, T.; Zhu, Y.; Cheong, S.W. Unfolding of Vortices into Topological Stripes in a Multiferroic Material. Phys. Rev. Lett. 2014, 112, 247601. [CrossRef] [PubMed]

27. Artyukhin, S.; Delaney, K.T.; Spaldin, N.A.; Mostovoy, M. Landau Theory of Topological Defects in Multiferroic Hexagonal Manganites. Nat. Mater. 2013, 13, 42-49. [CrossRef] [PubMed]

28. Poirier, M.; Laliberté, F.; Pinsard-Gaudart, L.; Revcolevschi, A. Magnetoelastic Coupling in Hexagonal Multiferroic $\mathrm{YMnO}_{3}$ Using Ultrasound Measurements. Phys. Rev. B 2007, 76, 174426. [CrossRef] 
29. Petit, S.; Moussa, F.; Hennion, M.; Pailhès, S.; Pinsard-Gaudart, L.; Ivanov, A. Spin Phonon Coupling in Hexagonal Multiferroic $\mathrm{YMnO}_{3}$. Phys. Rev. Lett. 2007, 99, 266604. [CrossRef] [PubMed]

30. Edwards, S.F.; Anderson, P.W. Theory of Spin Glasses. J. Phys. F Met. Phys. 1975, 5, 965. [CrossRef]

(C) 2018 by the authors. Licensee MDPI, Basel, Switzerland. This article is an open access article distributed under the terms and conditions of the Creative Commons Attribution (CC BY) license (http://creativecommons.org/licenses/by/4.0/). 